\title{
85643 - CARACTERIZAÇÃO DOS ATENDIMENTOS AOS IDOSOS E INTERVENÇÕES REALIZADAS PELO SERVIÇO AÉROMÉDICO
}

\author{
Apresentação Oral - Gerontologia
}

Anderson Abreu de Carvalho / Carvalho, AA / Universidade Federal de Santa Catarina; Aline Corrêa da Costa / Costa, AC / Universidade Federal de Santa Catarina; Keyla Cristiane do Nascimento / Nascimento, KC / Universidade Federal de Santa Catarina; Melissa Orlandi Honório Locks / Locks, MOH / Universidade Federal de Santa Catarina; Juliana Balbinote Reis Girondi / Gironde, JBR / Universidade Federal de Santa Catarina; Gustavo Lopes Soares / Soares, GL / Universidade Federal de Santa Catarina

Introdução: Com estilo de vida mais ativo, a população idosa acaba mais exposta aos fatores de riscos, ficando suscetíveis a doenças e acidentes, ocasionando os traumas(1). Esses tipos de demandas aumentam o atendimento de emergência para essa população(2). Pensando no atendimento pré-hospitalar, essa população exige, cuidados específicos e até mesmo intensivos por conta das suas peculiaridades. Objetivo: Analisar o perfil dos idosos e os cuidados de saúde realizados pelo serviço aeromédico do Batalhão de Operações Aéreas de Santa Catarina. Método: Pesquisa quantitativa, retrospectiva e transversal realizada entre novembro de 2017 a fevereiro de 2018 no Batalhão de Operações Aéreas de Santa Catarina. Os dados foram obtidos por meio do preenchimento de um instrumento, contendo variáveis como gênero, faixa etária, tipo de atendimento, procedimentos invasivos, entre outros. Foram incluídos os registros dos atendimentos realizados a pessoas com idade superior a 60 anos no período de 2013 a 2017. A análise dos dados deu-se pelo uso de estatística descritiva simples. Número do CEP: 2047148. Resultados: A amostra foi constituída por 538 atendimentos, sendo o maior número de atendimentos prestados ao sexo masculino $61,9 \%$, a média de idade geral foi 72,02. Teve prevalência os atendimentos realizados na cidade de Florianópolis $33,1 \%$. Na sua maioria nas residências $28,6 \%$. Prevaleceu os atendimentos classificados como agravos clínicos $63,76 \%$, sendo a Parada cardiorrespiratória a mais prevalente e as quedas o principal agravo das causas externas, representando 9,1\%. Em relação ao desfecho 73,05\% foram encaminhados a uma unidade hospitalar. Conclusão: Pode-se conhecer melhor o perfil dos atendimentos que foram prestados, identificando os principais cuidados de saúde que foram prestados durante a assistência pela equipe do aeromédico e o desfecho desses atendimentos.

Palavras-chave: Cuidado de Enfermagem. Idoso. Resgate aéreo.

Referências: 1. BRASIL, Ministério da Saúde. Estatuto do Idoso. 2013. Acesso em: 05 jun. 2017. 2. MIRANDA, Gabriella Morais Duarte; MENDES, Antônio da Cruz Gouveia; SILVA, Ana Lucia Andrade da. Population aging in Brazil: current and future social challenges and consequences. Revista Brasileira de Geriatria e Gerontologia, [s.l.], v. 19, n. 3, p.507-519, jun. 2016. FapUNIFESP (SciELO). http://dx.doi.org/10.1590/1809-98232016019.150140. 